SUPPORTING INFORMATION

\title{
Saturation of shear-induced isothermal crystallization of polymers at the steady-state and the entanglement-disentanglement transition
}

\author{
José A. Martins ${ }^{1,2}(*)$, Weidong Zhang ${ }^{1,2}$ and António M. Brito ${ }^{1}$ \\ (1) Departamento de Engenharia de Polímeros, Instituto de Polímeros e Compósitos, \\ Universidade do Minho, Campus de Azurém 4800-058 Guimarães PORTUGAL \\ $\left(^{2}\right)$ CICECO, Universidade de Aveiro, 3810-193 Aveiro, Portugal \\ Email: jamartins@dep.uminho.pt
}

Includes:

Additional information on the characterization of the steady-state

Additional information on the results of Figures $4 \mathrm{~b}$ and $6 \mathrm{~b}$

Evaluation of the Hamaker constant for polypropylene

References

Figures S1 and S2 


\section{Additional information on the characterization of the steady-state}

The purpose of the discussion below is to guarantee the reliability of the results obtained at the steady-state, namely the critical strains evaluated at its onset and the reptation time of critically sheared samples. For a specific sheared melt temperature, the variation of viscosity with time at constant shear rate, reaches the steady-state at a constant critical strain. For iPP at $220{ }^{\circ} \mathrm{C}$ it is $\approx 1970 \mathrm{~s} . u$. (Figure S1). The time at its onset for $6 \mathrm{~s}^{-1}$ is $\approx 330 \mathrm{~s}$, which is six times less the time needed for reaching the same state at $1 \mathrm{~s}^{-1}$. If sample degradation during the constant shearing had occurred for the long term experiments, it would be impossible to get the superposition shown.

To further test the latter assumption, the enthalpy of crystallization of unsheared and critically sheared samples was evaluated. The results of Figure S2 show comparable enthalpies of crystallization for both melt states. The relatively lower enthalpy measured for the sheared sample is a consequence of the memory effect of shear deformation on the overall crystallization kinetics, which was not fully erased during the melting at $210{ }^{\circ} \mathrm{C}$. This indicates that during the critical shearing, if any thermal degradation occurred, it had no significant effect on crystallization kinetics. Since thermal degradation is determined by dissociation of the weakest bonds, it may be predicted and experimentally evaluated. The thermal (non-oxidative) degradation of polypropylene has onset at $\approx 320^{\circ} \mathrm{C}$.

An additional test to validate the results obtained at the steady-state, and therefore its characterization, implies analyzing the reversibility of the process, so that the rheological behavior of the material, before and after rheological experiments at the steady-state, do not differ, within the experimental errors of the measurements, from the behavior recorded for unsheared samples. Figure S2b shows the flow curves, obtained in steady shear experiments, at $220^{\circ} \mathrm{C}$, for unsheared and critically sheared samples. Two different experiments were performed with these last samples. For the first one, after reaching the steady-state, the sample was cooled to room temperature, and further heated to $220{ }^{\circ} \mathrm{C}$, after which the steady-shear experiment was performed (triangles in Figure S2b). For the second one (circles), after reaching the steady-state, oscillatory shear experiments, described in Figure 3a, were performed at this state, after which the sample was cooled to room temperature, followed by the experimental procedure described previously. The

purpose of the experiment indicated by circles in Figure S2b was to ensure the validity of the 
oscillatory shear results obtained at the steady-state and the measurements of the reptation time for critically sheared samples, indicated in Figures 4b and 6b for polypropylene and polystyrene, respectively.

\section{Additional information on the results of Figures $4 b$ and $6 b$}

These two figures show the temperature variation of the reptation time for un-sheared and critically sheared samples. All oscillatory shear experiments were performed in the viscoelastic linear regime with a constant stress of $400 \mathrm{~Pa}$ for i-PP and $600 \mathrm{~Pa}$ for PS.

In particular, the results shown in Figure $4 d$ were repeated with two different rheometers. The average true sample temperature was evaluated from the nominal temperature of the

experiments following a temperature calibration procedure described previously. ${ }^{2}$ The dispersion in the results shown may be assigned to the use of different instruments and the effect that small temperature variations have on the value evaluated for the reptation time (despite the temperature calibration procedure mentioned above). For all oscillatory shear experiments performed with critically sheared samples, relatively high shear rates were used for reaching this state (5 s $\mathrm{s}^{-1}$ for i-PP and $10 \mathrm{~s}^{-1}$ for PS). After reaching this state (around 5 min after starting the experiment), oscillatory shear experiments were performed with the constant stresses mentioned above. The total time of an experiment used for evaluating the reptation time at the steady-state was around $30 \mathrm{~min}$.

The demonstration that the steady-state is a reversible state was made previously. This is shown clearly by the results of Figure S2b. This reversibility is only observed after a cooling to a temperature below the crystallization temperature or the glass transition temperature of the polymer.

\section{Evaluation of the Hamaker constant for polypropylene}

The Hamaker constant may be calculated from the Lifshitz theory of the van der Waals interactions, where the interacting forces are derived in terms of dielectric constants and refractive indexes. Since this theory is a continuum theory, strictly it should be used only when 
the separation of the chain molecules is larger than the molecular size. Nevertheless, it will be used here for estimating the Hamaker constant for polypropylene. Typical values of this constant for condensed phases (solid or liquid), interacting across vacuum, are $\approx 10^{-19} \mathrm{~J}$. When hydrocarbons are treated as an assembly of $\mathrm{CH}_{2}$ groups, the value obtained for it is around $5 \mathrm{x}$ $10^{-20} \mathrm{~J}$.

Considering two identical chains interacting across vacuum (which is usual in these conditions), the total Hamaker constant for the two chains contains an entropic zero-frequency contribution $\left(A_{v=0}\right)$, which includes the Keesom and Debye dipolar contributions to the van der Waals interactions, and a dispersion energy contribution $\left(\mathrm{A}_{v>0}\right)$, which includes the London energy contribution. If the interactions are assumed to be non-retarded and additive, the result obtained is ${ }^{3}$

$$
A_{\text {total }}=A_{v=0}+A_{v>0}=\frac{3}{4} k_{B} T\left(\frac{\varepsilon-\varepsilon_{o}}{\varepsilon+\varepsilon_{o}}\right)^{2}+\frac{3 h v_{e}}{16 \sqrt{2}} \cdot \frac{\left(n^{2}-n_{o}^{2}\right)^{2}}{\left(n^{2}+n_{o}^{2}\right)^{3 / 2}},
$$

where $\varepsilon$ and $\varepsilon_{o}$ are the static dielectric constants for the polymer and vacuum, $n$ and $n_{\mathrm{o}}$ are the corresponding refractive indexes, $h$ and $k_{\mathrm{B}}$, the Planck and Boltzmann constants, respectively, $T$ the absolute temperature and $v_{\mathrm{e}}$ the main electronic absorption frequency in the UV $\left(\cong 3.0 \times 10^{15}\right.$ $\mathrm{Hz})$.

For hydrocarbons $\rho \approx 3.3 \times 10^{28} \mathrm{~m}^{-3}$ per $\mathrm{CH}_{2}$ group. The molecular diameter, evaluated at $T=473 \mathrm{~K}$, from the van der Waals volume of polypropylene $\left(30.7 \mathrm{~cm}^{3} / \mathrm{mol}\right)$ and its molar thermal expansivity $\left(3.07 \times 10^{-2} \mathrm{~cm}^{3} / \mathrm{Kmol}\right)$ was $5.57 \AA .^{1}$ At $T=473 \mathrm{~K}$, with $\varepsilon=2.2$ and $n=1.5$, the value of the Hamaker constant for two polypropylene chains interacting across vacuum is $7.1 \times 10^{-20} \mathrm{~J}$.

\section{References}

(1) Van Krevelen, D. W. Properties of Polymers; $3^{\text {rd }}$ edition Elsevier Science B. V.: Amsterdam, 1997.

(2) Martins, J. A.; Zhang, Wd.; Carvalho, V.; Brito, A. M.; Romero, M.; Soares, F. O. Polymer 2003, 44, 8071

(3) Israelachvili, J. Intermolecular and Surface Forces, Academic Press Limited, London, 1992. 


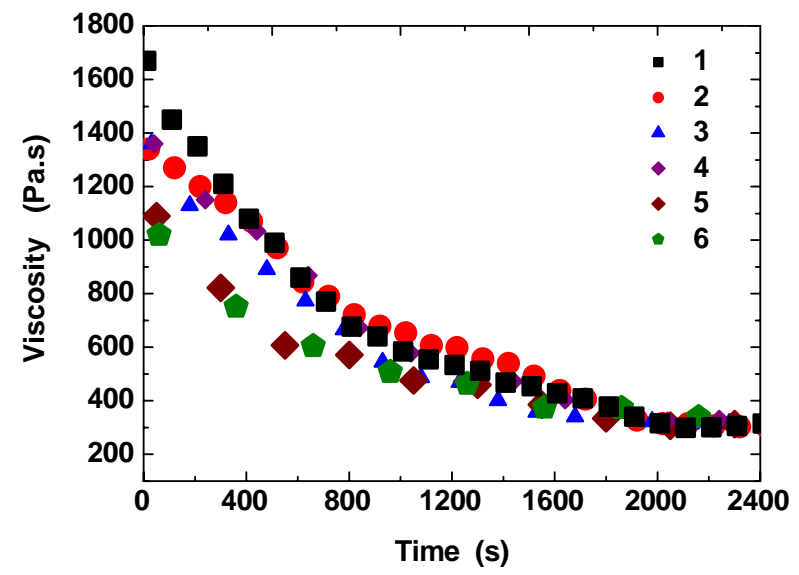

Figure S1. Time-shear rate superposition of time dependent viscosity. Stress growth experiments of iPP at $220^{\circ} \mathrm{C}$ for the constant shear rates indicated. The curve at $1 \mathrm{~s}^{-1}$ was selected for reference. The shift factor of the results at a shear rate $\dot{\gamma}$ with respect to reference shear rate $\dot{\gamma}_{0}$ is $a_{\dot{\gamma} / \dot{\gamma}_{0}}=t_{\dot{\gamma}} / t_{\dot{\gamma}_{o}}=\dot{\gamma}_{o} / \dot{\gamma}$. 

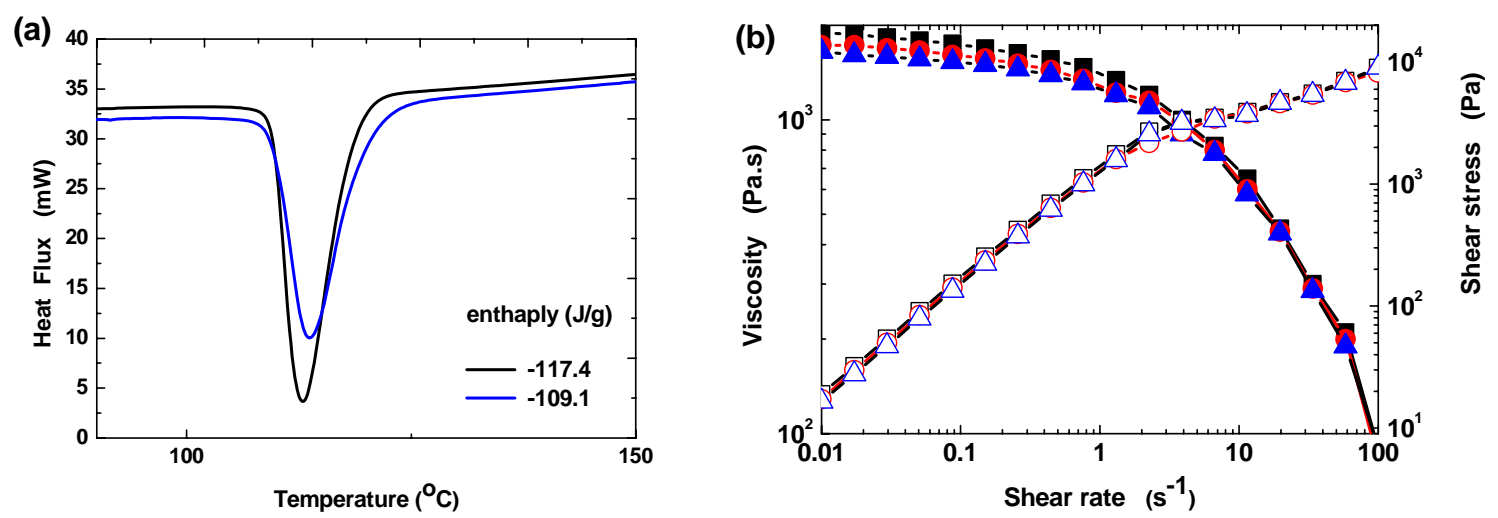

Figure S2. Evaluation of critical strain effects on crystallization and flow behavior of critically sheared samples. (a) DSC cooling scans at $-10{ }^{\circ} \mathrm{C} / \mathrm{min}$ for un-sheared (solid line) and critically sheared (dotted line) iPP at $210^{\circ} \mathrm{C}$, with a shear rate of $2 \mathrm{~s}^{-1}$, after saturation of shear (1400 s). All samples were previously heated up to $210^{\circ} \mathrm{C}$. The enthalpies of crystallization were measured between $160{ }^{\circ} \mathrm{C}$ and $85^{\circ} \mathrm{C}$. (b) Shear viscosity (full symbols) and shear stress (open symbols) variation with shear rate for un-sheared (squares) and critically sheared samples (triangles) at 220 ${ }^{\circ} \mathrm{C}$. These samples were previously cooled to room temperature. Results indicated by circles also refer to critically sheared samples but, preceding the cooling, the sample was submitted to an oscillatory shear experiment at the steady-state. 